\title{
TAREFAS DE DESENVOLVIMENTO E CRITÉRIOS DE COMPETÊNCIA EM ADOLESCENTES E JOVENS ADULTOS
}

\author{
Maria João Carapeto \\ Universidade de Evora, Escola de Ciencias Sociais, Departamento de Psicologia mjoaocarapeto@gmail.com \\ Heldemerina Pires \\ CIEP-UE - Centro de Investigação em Educação e Psicologia - Universidade de Évora, Portugal \\ Universidade de Evora, Escola de Ciencias Sociais, Departamento de Psicologia \\ hsp@uevora.pt \\ Luísa Grácio \\ CIEP-UE - Centro de Investigação em Educação e Psicologia - Universidade de Évora, Portugal \\ Universidade de Evora, Escola de Ciencias Sociais, Departamento de Psicologia \\ mlg@uevora.pt
}

Recepción Artículo: 13 octubre 2021

Admisión Evaluación: 13 octubre 2021

Informe Evaluador 1: 13 octubre 2021

Informe Evaluador 2: 14 octubre 2021

Aprobación Publicación: 14 octubre 2021

\section{RESUMO}

A construção da identidade e de relações de intimidade ou o casamento, entre outras, têm sido consideradas importantes tarefas de desenvolvimento normativo para adolescentes e jovens adultos, respetivamente. É consensualmente aceite que os critérios para um desenvolvimento adaptativo podem ser diferentes em diferentes momentos históricos, sociedades e culturas, e que diferentes pessoas podem entender de modo diverso o seu próprio desenvolvimento ou o dos outros. Neste contexto, o objetivo geral deste estudo foi 0 de explorar os critérios de competência utilizados pelos indivíduos para descrever adolescentes e jovens adultos da sua comunidade. Um questionário foi aplicado a uma amostra de estudantes universitários de 1ํano para que indicassem 3-4 descritores de 2 adolescentes e 2 jovens adultos seus conhecidos que considerassem estar a viver bem a etapa do desenvolvimento em que se encontram. Foram reunidos 449 descritores cujo conteúdo foi submetido a análise de conteúdo, depois combinando abordagem qualitativa e quantitativa. Os descritores foram codificados nas categorias de competências académicas, comportamento positivo, relações positivas com a família e com pares, caraterísticas de personalidade, construção da identidade, afetividade, atividades e trabalho, e relações amorosas e nova família. Verificou-se que os critérios de competência mais frequentes foram, para os adolescentes, a construção da identidade, o comportamento positivo e as competências académicas, e para os jovens adultos, atividades/trabalho, construção da identidade e comportamento positivo. Os descritores de competências académicas e relações positivas com a família foram mais frequentemente usadas para os adolescentes do que para os jovens adultos. A construção da identidade e as relações amorosas e nova família foram mais frequente- 


\section{TAREFAS DE DESENVOLVIMENTO E CRITÉRIOS DE COMPETÊNCIA EM ADOLESCENTES E JOVENS ADULTOS}

mente referidas para os jovens adultos. Este estudo sugere alguma continuidade entre as conceções leigas de competência para adolescentes e jovens adultos bem como a emergência de critérios mais específicos de cada etapa do desenvolvimento.

Palavras chave: tarefas de desenvolvimento; competência; conceções leigas; identidade; estudantes universitários

\section{ABSTRACT}

Developmental tasks and competence criteria for adolescents and young adults. The construction of identity and intimate relationships or marriage, among others, have been considered important normative developmental tasks for adolescents and young adults, respectively. It is generally accepted that the criteria for adaptive development can be different in different historical moments, societies and cultures, and that different people can understand their own development differently and that of others. In this context, the general objective of this study was to explore the competence criteria used by individuals to describe adolescents and young adults in their communities. A questionnaire was administered to a sample of first-year university students to indicate 3-4 descriptors of 2 teenagers and 2 young adults they knew and considered to be doing well at their stage of development. A total of 449 descriptors was collected, whose content was submitted to content analysis, then combining qualitative and quantitative approaches. The descriptors were coded in the categories of academic competence, positive conduct, positive relationships with family and peers, personality characteristics, identity construction, affect, activities and work, and romantic relationships and new family. It was found that the most frequent competence criteria were, for adolescents, identity construction, positive conduct and academic competence, and for young adults, activities/work, identity construction and positive conduct. The descriptors of academic competence and positive relationships with the family were used more often for adolescents than for young adults. The construction of identity and romantic relationships and new family were most frequently mentioned for young adults. This study suggests some continuity between lay conceptions of competence for adolescents and young adults, as well as the emergence of more specific criteria for each stage of development.

Keywords: developmental tasks; competence; lay conceptions; identity; college students

\section{INTRODUÇÃO}

0 conceito de tarefa de desenvolvimento tem sido crucial para o estudo e compreensão dos processos de desenvolvimento de adolescentes e jovens adultos, bem como ao longo de todo o ciclo de vida. Uma tarefa de desenvolvimento pode ser definida como uma tarefa "which arises at or about a certain period in the life of an individual, successful achievement of which leads to happiness and to success with later tasks, while failure leads to unhappiness in the individualy... and difficulty with later tasks" (Havighurst, 1948, p. 2). Havighurst (1948), que inicialmente propôs o conceito, identificou, como tarefas de desenvolvimento da adolescência (12-18 anos), desenvolver relações íntimas com pares de ambos os sexos, adotar um papel social feminino ou masculino, aceitar 0 seu corpo, alcançar independência emocional dos pais, preparar-se para o mundo profissional, preparar-se para o casamente e vida familiar, construir um sistema de valores e ética que guie o comportamento, e apresentar comportamento socialmente responsável. Uma vez realizadas estas tarefas os indivíduos estariam prontos para lidar com as tarefas destinadas aos jovens adultos (18-30 anos), designadamente iniciar uma carreira no mundo do trabalho, desenvolver uma relação estável e aprender a viver com o parceiro/a, construir uma família própria e aprender a gerir a casa de modo independente, cuidar da família, assumir responsabilidades cívicas e integrarse num grupo social. De modo algo semelhante, Erikson (1959) propôs que o desenvolvimento psicossocial resulta da combinação entre a maturação do indivíduo com os desafios que a sociedade the apresenta em cada etapa do ciclo de vida, e da resolução das crises ou conflitos com que assim são confrontados. A construção da identidade é a solução adaptativa da crise (ou tarefa desenvolvimental) central da adolescência e a construção de relações íntimas a solução adaptativa da crise normativa dos jovens adultos.

Mais recentemente, Masten \& Tellegen (2012) definiram tarefas de desenvolvimento como "the accomplish- 
ments expected within a given society or culture in historical context for people during different age periods over the life course" (p. 348). Segundo os autores, essas expetativas são, afinal, os critérios que servem de referência para os membros da sociedade (e os próprios) avaliarem os progressos dos indivíduos e a sua competência. Estas realizações que 0 indivíduo exibe ao desenvolver-se correspondem, afinal, ao conceito de competência, "a pattern of effective performance in the environment, evaluated from the perspective of development in ecological and cultural context" (Masten \& Coatsworth, 1995, ref. Masten \& Tellegen, 2012, p. 348).

Neste contexto, torna-se crucial identificar os critérios socioculturais de competência para cada período do ciclo de vida (e.g., Durbrow et al, 2001; Masten \& Tellegen, 2012), tal como são percebidos pelos próprios membros de cada comunidade. Durbrow e colegas (2001), por exemplo, estudaram as conceções de competência de mães vivendo em contexto de pobreza nas Filipinas, ilha de S. Vicente (Caraíbas) e nos Estados Unidos da América, e constataram que as crianças e adolescentes competentes eram descritos como bem comportados e obedientes, estudantes satisfatórios, que ajudam a família e são amigáveis com os pares. Além disso, as mães filipinas raramente indicavam rapazes adolescentes como exemplo de crianças competentes. Estes autores equacionaram competência /tarefas de desenvolvimento como os aspetos da vida que parecem funcionar como critérios para ajuizar se uma pessoa está a "caminhar bem" (doing wel/) na sua etapa da vida e na comunidade em que se encontra.

Coloca-se assim a questão de reavaliar a validade das tarefas normativas de desenvolvimento que decorrem das teorias clássicas, especificamente no contexto sociocultural português e, ainda mais, tendo em consideração que vivemos tempos históricos diferentes daqueles em que tais propostas teóricas foram apresentadas. Por exemplo, nas sociedade ocidentais ditas desenvolvidas em que a educação se estende e a entrada no mundo do trabalho se empurra para mais adiante no ciclo de vida, é comummente aceite que a adolescência se prolonga até meados da década dos 20 anos de idade (Sawyer et al., 2018) ao mesmo tempo que outros autores afirmam que se está a definir um novo período do desenvolvimento entre a adolescência e a etapa de jovens adultos, designadamente a idade adulta emergente (emerging adulthood; Arnett, 2000, 2016). Alguns estudos mostram mesmo que as tarefas previstas pela teoria são cumpridas por cada vez menos jovens adultos, inclusive em Portugal (Sousa \& Fonseca, 2014).

Em suma, no estado atual do conhecimento torna-se importante perceber quais são as competências consideradas importantes para descrever os adolescentes e jovens adultos que apresentam um funcionamento positivo, do ponto de vista dos próprios portugueses na atualidade. Este estudo procura oferecer um contributo para esse esclarecimento.

\section{OBJETIVOS DA INVESTIGAÇÃO}

Identificar os critérios de competência esperados para adolescentes e jovens adultos portugueses, do ponto de vista de pessoas que vivem em Portugal, e compará-los com as tarefas de desenvolvimento tradicionalmente propostas para estes dois períodos do desenvolvimento.

\section{METODOLOGIA}

\section{Participantes}

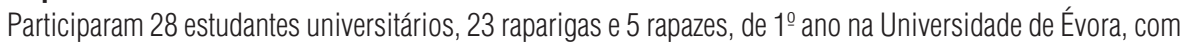
idades entre os 18 e os 25 anos de idade, $M=19.57, \mathrm{DP}=1.83$.

\section{Instrumento}

Foi elaborado um questionário baseado na entrevista de Criteria of Child Competence Interview (Durbrow et al., 2001), com administração oral das instruções e questões. Num primeiro momento, os participantes foram instruídos para identificar uma pessoa concreta dos seus conhecimentos que se encontrasse na adolescência e estivesse a viver bem essa etapa da sua vida (o indivíduo de referência), e depois para registarem, numa folha 


\section{TAREFAS DE DESENVOLVIMENTO E CRITÉRIOS DE COMPETÊNCIA EM ADOLESCENTES E JOVENS ADULTOS}

preparada para o efeito, a idade e género dessa pessoa, bem como 3-4 caraterísticas que Ihe indicassem que esse adolescente estava de facto a viver bem essa etapa da sua vida. 0 processo foi repetido para outro adolescente do género oposto, e repetido depois para obter descritores de dois jovens adultos de géneros diferentes. Cada participante forneceu assim um conjunto de descritores (ou critérios de competência) de 4 indivíduos de referência, designadamente dois adolescentes (géneros feminino e masculino) e de dois jovens adultos (idem), bem como as respetivas idades (recolheram-se também dados relativos a pessoas meia idade e idosos, que não foram considerados neste estudo).

\section{Procedimentos}

Obtido, por escrito, o consentimento informado dos participantes, o questionário foi aplicado na primeira aula de uma unidade curricular de psicologia do desenvolvimento. Uma vez distribuída a folha para registo das respostas individuais, as instruções foram sendo proferidas em voz alta, dando tempo aos participantes para registar a sua resposta a cada passo.

A análise de conteúdo das respostas (Bardin, 2008) desenrolou-se em três fases: (a) Pre-analise: cada participante recebeu um código de identificação e os descritores foram transcritos para um ficheiro de texto, separadamente por participante e por etapa do desenvolvimento e género do indivíduo de referência. Uma leitura prévia foi realizada por duas autoras que, depois, de modo consensual, consideraram ser suscetíveis de ser codificados em categorias similares aos domínios de competência encontrados por Durbrow et al. (2001) para adolescentes (i.e., competências académicas, comportamento positivo, relações positivas com a família e com os pares) e em acrescentar outras categorias (i.e., caraterísticas de personalidade, construção da identidade, afetividade, atividades e trabalho, relações amorosas e nova família) (Tabela 1). Foi também incluída uma categoria de "outros" para acolher os descritores que não pudessem ser classificados nas categorias propostas. Concordaram também quanto à delimitação das unidades de registo. (b) Exploracao do material: os descritores foram codificados nas 10 categorias, de modo independente, pelas referidas duas autoras, considerando criterios de inclusão, exclusao e relevancia mutua, tendo sido possível, no final, chegar a acordo quanto à codificação de todas as unidades de registo. (c) Análise quantitativa: procedeu-se a contagem da frequencia dos descritores em cada categoria e, com a ajuda do IBM SPSS (versão 24.0), foram calculadas as estatísticas descritivas (frequências, médias e desvio-padrão) de cada categoria, para cada etapa do desenvolvimento, e realizados testes não paramétricos (teste de Wilcoxon) para comparar as frequências de cada categoria na adolescência e nos jovens adultos.

\section{RESULTADOS}

Foram obtidos 209 descritores de 56 adolescentes, com idade que variou, para o género feminino, entre os 13 e os 20 anos de idade, $M=16, S D=1.87$, e, para o género masculino, entre os 12 e os $19, M=15.96, S D=$ 2.01. Obtiveram-se também 240 descritores de 56 jovens adultos, com idade, no género feminino, entre 18 e 44 anos, $\mathrm{M}=24.18, \mathrm{DP}=4.94$, e, no género masculino, entre 19 e 34 anos, $\mathrm{M}=23.32, \mathrm{DP}=3.30$.

Como mostra a Tabela 2, os descritores relativos à construção da identidade são os mais frequentemente usados para descrever a competência dos adolescentes (16\%), seguindo-se o comportamento positivo (14\%) e as competências académicas (13\%), enquanto para os jovens adultos são mais frequentes as atividades e trabaIho (21\%), seguindo-se a construção da identidade (21\%) e o comportamento positivo (16\%). As relações amorosas e a construção de nova família nunca são mencionadas para descrever adolescentes, tema que surge para descrever a competência dos jovens adultos embora com uma das mais baixas frequências (5\%) na Tabela 2. 
Tabela 1

Categorias, critérios de codificação, e exemplos dos descritores de adolescentes e jovens adultos

\begin{tabular}{|c|c|c|}
\hline Categorias & Critérios & Exemplos \\
\hline $\begin{array}{l}\text { Competências } \\
\text { académicas }\end{array}$ & $\begin{array}{l}\text { Bons resultados escolares. } \\
\text { Satisfação com a } \\
\text { escola/universidade. Valorizar a } \\
\text { educação. Adaptação a transições } \\
\text { no sistema educativo. Formação. } \\
\text { Estudos concluídos. }\end{array}$ & $\begin{array}{l}\text { Ad1 - "boa aluna"; Ad14 - "boa interação } \\
\text { com os professores"; JA14 - "está muito } \\
\text { feliz com a sua licenciatura" }\end{array}$ \\
\hline $\begin{array}{l}\text { Comportamento } \\
\text { positivo }\end{array}$ & $\begin{array}{l}\text { Comportamento prossocial, } \\
\text { responsável ou socialmente } \\
\text { recomendado. }\end{array}$ & $\begin{array}{l}\text { Ad4 - "respeita as ordens que lhe são } \\
\text { dadas"; Ad23 - "responsável"; JA3 - } \\
\text { "trabalhadora" }\end{array}$ \\
\hline $\begin{array}{l}\text { Relações } \\
\text { positivas com a } \\
\text { família }\end{array}$ & $\begin{array}{l}\text { Ligação positiva ou apoio familiar } \\
\text { relativamente à família de origem. } \\
\text { Satisfação com a família de } \\
\text { origem. }\end{array}$ & $\begin{array}{l}\text { Ad10 - "tem uma boa relação com os pais"; } \\
\text { JA2 - "uma família com quem pode contar } \\
\text { para o que der e vier"; JA4 - "muito ligada à } \\
\text { família" }\end{array}$ \\
\hline $\begin{array}{l}\text { Relações } \\
\text { positivas com } \\
\text { pares }\end{array}$ & $\begin{array}{l}\text { Ter amigos. Passar tempo com os } \\
\text { amigos. Satisfação com relações de } \\
\text { amizade. }\end{array}$ & $\begin{array}{l}\text { Ad3 - "tem um círculo de amigos"; Ad27 - } \\
\text { "faz amigos com facilidade"; JA1 - "amiga } \\
\text { do seu amigo" }\end{array}$ \\
\hline $\begin{array}{l}\text { Caraterísticas de } \\
\text { personalidade }\end{array}$ & $\begin{array}{l}\text { Caraterísticas de personalidade e } \\
\text { nas relações interpessoais. }\end{array}$ & $\begin{array}{l}\text { Ad14 - "extremamente extrovertido"; JA1 - } \\
\text { "corajosa"; JA22 - "resiliente" }\end{array}$ \\
\hline $\begin{array}{l}\text { Construção da } \\
\text { identidade }\end{array}$ & $\begin{array}{l}\text { Sentido de ser único, confiante. } \\
\text { Sentido de orientação para o futuro, } \\
\text { ter objetivos. Autonomia, } \\
\text { independência (qualquer domínio). } \\
\text { Crescimento pessoal. }\end{array}$ & $\begin{array}{l}\text { Ad2 - "tem as metas definidas e é } \\
\text { trabalhadora de modo a tentar alcançá-las"; } \\
\text { Ad11 - "autonomia em algumas decisões"; } \\
\text { JA11 - "consegue ter uma vida } \\
\text { independente" }\end{array}$ \\
\hline Afetividade & $\begin{array}{l}\text { Afetos predominantes. Estabilidade } \\
\text { ou autorregulação emocional. Bem- } \\
\text { estar emocional. }\end{array}$ & $\begin{array}{l}\text { Ad6 - "divertida, sempre feliz"; JA2 - "tem } \\
\text { sempre um sorriso na cara"; JA7 - } \\
\text { "estabilidade emocional" }\end{array}$ \\
\hline $\begin{array}{l}\text { Atividades e } \\
\text { trabalho }\end{array}$ & $\begin{array}{l}\text { Atividades extracurriculares e de } \\
\text { tempos livres, desporto. Trabalho } \\
\text { remunerado. Satisfação com } \\
\text { atividades e trabalho. Capacidade } \\
\text { para conciliar diversas atividades. }\end{array}$ & $\begin{array}{l}\text { Ad7 - "organiza a vida e os seus horários, } \\
\text { tendo tempo para tudo" } \\
\text { Ad15 - "pratica o seu desporto favorito" } \\
\text { JA8 - "está a ter sucesso a nível } \\
\text { profissional" }\end{array}$ \\
\hline $\begin{array}{l}\text { Relações } \\
\text { amorosas e nova } \\
\text { família }\end{array}$ & $\begin{array}{l}\text { Relações românticas, namoro, } \\
\text { casamento; estabilidade nessas } \\
\text { relações. Construir a sua própria } \\
\text { família, ter filhos. }\end{array}$ & $\begin{array}{l}\text { JA8 - "relação estável"; JA11 - "começa a } \\
\text { pensar casar e em constituir família" } \\
\text { JA23 - "já tem filhos" }\end{array}$ \\
\hline Outros & $\begin{array}{l}\text { Saúde e bem-estar. Aspetos } \\
\text { financeiros. Outros não passíveis } \\
\text { de codificação noutras categorias. }\end{array}$ & $\begin{array}{l}\text { Ad4 - "tem uma boa alimentação"; Ad5 - } \\
\text { "esperto"; JA18 - "pergunta pelos outros" }\end{array}$ \\
\hline
\end{tabular}

Verifica-se também uma distribuição mais homogénea das frequências de todas as categorias quanto aos descritores da adolescência, com frequências entre 7\% e 16\% (exceto o caso das relações amorosas e nova família, que teve frequência nula); pelo contrário, os descritores dos jovens adultos tendem a concentrar-se em algumas categorias, como as mencionadas acima, e deixam outras categorias com frequências relativamente mais baixas, como é o caso das categorias relativas a relações com outros (com a família, 4\%; relações amorosas e nova família, $5 \%$; com pares, $5 \%$ ). 


\section{TAREFAS DE DESENVOLVIMENTO E CRITÉRIOS DE COMPETÊNCIA EM ADOLESCENTES E JOVENS ADULTOS}

\section{Tabela 2}

Estatísticas descritivas das categorias mencionadas pelos participantes $(N=28)$ para descrever 56 adolescentes e 56 jovens adultos que caminham bem na sua etapa do desenvolvimento

\begin{tabular}{|c|c|c|c|c|c|c|c|c|}
\hline \multirow[t]{2}{*}{ Categoria } & \multicolumn{4}{|c|}{ Adolescentes } & \multicolumn{4}{|c|}{ Jovens adultos } \\
\hline & Freq & $\%$ & $\mathrm{M}$ & $\mathrm{DP}$ & Freq & $\%$ & $\mathrm{M}$ & $\mathrm{DP}$ \\
\hline $\begin{array}{l}\text { Competências } \\
\text { académicas }\end{array}$ & 27 & 12.92 & .96 & .84 & 11 & 4.58 & .39 & .88 \\
\hline $\begin{array}{l}\text { Comportamento } \\
\text { positivo }\end{array}$ & 30 & 14.35 & 1.07 & 1.56 & 38 & 15.83 & 1.36 & 1.64 \\
\hline $\begin{array}{l}\text { Relações } \\
\text { positivas com a } \\
\text { família }\end{array}$ & 23 & 11.00 & .82 & .94 & 9 & 3.75 & .32 & .67 \\
\hline $\begin{array}{l}\text { Relações } \\
\text { positivas com } \\
\text { pares }\end{array}$ & 21 & 10.05 & .75 & .75 & 13 & 5.42 & .46 & .84 \\
\hline Personalidade & 20 & 9.57 & .71 & .85 & 23 & 9.58 & .82 & 1.16 \\
\hline $\begin{array}{l}\text { Construção da } \\
\text { identidade }\end{array}$ & 33 & 15.79 & 1.18 & 1.19 & 50 & 20.83 & 1.79 & 1.20 \\
\hline Afetividade & 20 & 9.57 & .71 & .90 & 24 & 10.00 & .86 & .76 \\
\hline $\begin{array}{l}\text { Atividades e } \\
\text { trabalho }\end{array}$ & 20 & 9.57 & .71 & .71 & 51 & 21.25 & .96 & 1.17 \\
\hline $\begin{array}{l}\text { Relações } \\
\text { amorosas e nova } \\
\text { família }\end{array}$ & 0 & 0.00 & .00 & .00 & 11 & 4.58 & .39 & .74 \\
\hline Outros & 15 & 7.18 & .69 & .79 & 10 & 4.17 & .36 & .73 \\
\hline Total & 209 & 100.00 & & & 240 & 100.00 & & \\
\hline
\end{tabular}

Nota. Freq - Frequência, M - Média, DP - Desvio padrão.

Por fim, são mais frequentemente referidas para descrever os adolescentes do que os jovens adultos, as competências académicas, $z=-2,369, p=.018$, e relações positivas com a família, $z=-2.828, p=.005$, e mais frequentemente usadas para descrever os jovens adultos a construção da identidade, $z=-2.060, p=.039$, e relações amorosas e nova família, $z=-2.636, p=.008$. Nas restantes categorias não se verificam diferenças significativas na frequência dos descritores das duas etapas do desenvolvimento.

\section{DISCUSSÃO}

Este estudo pretendia contribuir para esclarecer quais os mais relevantes critérios de competência para descrever adolescentes e jovens adultos portugueses, do ponto vista de pessoas das suas comunidades. Pretendia também compará-Ios com as tarefas de desenvolvimento tradicionalmente propostas para estes dois períodos do desenvolvimento. Os resultados sugerem semelhanças entre as abordagens dos nossos participantes e a teoria, mas assinalam também algumas diferenças.

Em primeiro lugar, globalmente, os critérios de competência que emergem são compatíveis com as tarefas de desenvolvimento apresentadas, na literatura, para a adolescência e idade adulta (Arnett, 2000, 2016; Erikson, 1959; Havighurst, 1948).

Na adolescência distingue-se a importância do sucesso escolar, possivelmente como etapa para a construção de uma carreira profissional futura, da construção da identidade (e.g., comprometer-se com objetivos) (Erikson, 1959; Meeus, 2011) e da autonomia (Smetana et al., 2014); do comportamento positivo como comportamento de natureza prossocial e positivamente sancionado pela sociedade (responsável, ajudar os outros, etc.) (Havighurst, 1948); das relações de amizade e vida social ativa (Smetana et al., 2014). 
Além disso, os participantes referem também as relações positivas com a família, mais frequentemente relativas ao apoio familiar que oferecem ao adolescente (mas nunca mencionam apoio que 0 adolescente ofereça à família, ao contrário do que evidencia 0 estudo de Durbrow et al., 2001, em comunidades pobres). Este critério de competência é interessante, porque, à primeira vista, não assenta numa habilidade própria dos adolescentes considerados como indivíduos separados, mas antes inclui-os numa unidade social competente, a família. São também indicadas atividades de natureza extracurricular e desportiva (e habilidades de organização do tempo), que não são referidas em estudos anteriores, mas que possivelmente são percebidas como fazendo parte de uma melhor preparação para uma vida profissional futura que se vislumbra competitiva (Carapeto, 2019a).

Verificou-se a ausência, surpreendente, de referências a relações românticas e namoro na adolescência, contrariando a expetativa desta ser uma etapa de estabelecimento das primeiras relações amorosas (Smetana et al., 2014). Pode ser explicado pela maior valorização e centralidade da componente escolar dos adolescentes na sociedade portuguesa, em cujo contexto as relações amorosas parecem ser um distrator ou se afiguram como um risco e não tanto como uma competência. Esta explicação faz sentido tanto mais que os participantes foram estudantes universitários que chegaram recentemente à universidade e recentemente tiveram de lidar, de modo intenso, com a exigência académica dos exames de conclusão do ensino secundário em Portugal e o sistema de acesso ao ensino superior.

Os jovens adultos competentes são descritos por possuírem carreiras profissionais já em curso e estabilidade profissional, como proposto pelas abordagens clássicas do desenvolvimento (Havighurst, 1948; Sousa \& Fonseca, 2014). A construção da identidade continua a ser um tema central da competência nesta etapa do desenvolvimento, assim apoiando as caraterísticas propostas para o período da adultez emergente (Arnett, 2000, 2016) e outras perspetivas que colocam a construção da identidade como um tema importante ao longo de todo o ciclo de vida (Erikson, 1959; McAdams, 2006). 0 comportamento e envolvimento cívico (e.g., responsabilidade) continua, possivelmente de modo distinto, a ser cuma competência importante nesta etapa da vida, aliás na linha das abordagens tradicionais das tarefas de desenvolvimento (Havighurst, 1948).

Comparando diretamente as competências de adolescentes e jovens adultos, as competências académicas são mais referidas como critério de desenvolvimento adaptativo para os adolescentes do que para os jovens adultos, tal como esperado. Todavia, parece que a educação e formação profissional ainda em curso carateriza ainda uma parte dos jovens adultos, por ventura os mais novos entre os referidos e/ou que optaram por estudos universitários (porventura mais longos).

Embora não desaparecendo as referências à família de origem e ao apoio que esta oferece, tão centrais na descrição dos adolescentes competentes, esta temática perde claramente terreno nos jovens adultos, que, no entanto, adicionam temas relacionados com outros contextos relacionais, ausentes na adolescência, como as relações românticas, bem como construção de uma nova família, uma diferença esperada de acordo com as tarefas de desenvolvimento propostas na literatura (Erikson, 1959; Havighurst, 1948).

A construção da identidade, embora uma das mais mencionadas competências em cada uma das etapas do desenvolvimento, recebe um volume significativamente maior entre os descritores dos jovens adultos. Dá assim conta da importância central crescente deste tema no desenvolvimento na adolescência como ainda da sua possível intensificação nos jovens adultos (Arnett, 2000, 2016), possivelmente em associação aos objetivos e compromissos relacionados com as relações amorosas e no contexto do trabalho (Meeus, 2011). Nos adolescentes, a construção da identidade aparece mais frequentemente associada ou na contiguidade de conteúdos de natureza académica e da gestão de múltiplas atividades formativas. Outro aspeto que vem reforçar a importância dos temas da construção da identidade nos jovens adultos, além do maior numero de descritores, é a maior concentração dos descritores num número mais reduzido de categorias, relativamente aos adolescentes. Por outro lado, a maior dispersão dos descritores pelas categorias que descrevem os adolescentes pode estar relacionada com 0 seu envolvimento múltiplas e diversas transições, ou tarefas de desenvolvimento (Carapeto, 2019a, 2019b; Coleman, 2011). 


\section{TAREFAS DE DESENVOLVIMENTO E CRITÉRIOS DE COMPETÊNCIA EM ADOLESCENTES E JOVENS ADULTOS}

Por outro lado, em ambas as etapas há categorias com uma presença comum e semelhante, em termos de frequências, em ambas as etapas do desenvolvimento. É o caso da afetividade positiva e estabilidade emocional, que contraria a ideia de a adolescência ser o período normativo de agitação e tensão que alguns têm defendido (Arnett, 1999; Hollenstein \& Lougheed, 2013) e que acrescenta o bem-estar subjetivo como critério de competência de adolescentes e jovens adultos (Diener, 2000; Sousa \& Fonseca, 2014). As caraterísicas de personalidade foram usadas também indistintamente para descrever jovens das duas etapas, possivelmente sem ligação com especificidades da etapa do desenvolvimento.

\section{CONCLUSÕES}

Este estudo mostra semelhanças entre as competências definidoras da adolescência e dos jovens adultos indicadas na literatura e as percebidas pelos participantes (e.g., construção da identidade na adolescência e início da carreira profissional nos jovens adultos), Mostra também distintos critérios de competência na adolescência (e.g., académicas) e nos jovens adultos (e.g., relações amorosas e construção de uma nova família), ao mesmo tempo que sugere competências idênticas nas duas fases (e.g., construção da identidade) ou dimensões que de modo transversal caraterizam adolescentes e jovens adultos competentes na sua etapa do desenvolvimento (e.g., afetividade positiva e certas caraterísticas de personalidade).

Os resultados obtidos encorajam estudos futuros com amostras mais diversificadas relativamente a caraterísticas sociodemográficas, ou métodos que possibilitem aprofundar a perspetiva dos sujeitos (ex., entrevista) e resultados quantitativos. Este estudo oferece um pequeno contributo para a compreensão das expetativas sociais sobre a competência dos jovens na sociedade portuguesa, e tem implicações para a prática em psicologia e educação, já que estas expetativas, socialmente partilhadas, moldam possivelmente as experiências formativas disponibilizadas aos jovens de hoje para serem os adultos de amanhã. Por exemplo, a pressão para a obtenção dos melhores resultados escolares pode estar associada aos preocupantes níveis de stresse relacionado com escola reportado por adolescentes portugueses noutros estudos (Inchley \& Currie, 2016).

\section{FINANCIAMENTO}

Este trabalho é financiado por fundos nacionais através da FCT - Fundação para a Ciência e a Tecnologia, I.P., no âmbito do projeto UIDB/04312/2020

\section{REFERÊNCIAS BIBLIOGRÁFICAS}

Arnett, J. J. (1999). Adolescent storm and stress, reconsidered. American Psychologist, 54, 317-326. doi:10.1037/0003-066X.54.5.317

Arnett, J. J. (2000). Emerging adulthood: A theory of development from the late teens through the twenties. American psychologist, 55(5), 469-480.

Arnett, J. J. (2016). Does emerging adulthood theory apply across social classes? National data on a persistent question. Emerging adulthood, 4(4), 227-235.

Bardin, L. (2008). Analise de conteudo (5th Ed.). Lisboa: Edicoes 70.

Carapeto, M. J. (2019a). Desenvolvimento na adolescência e aprendizagem ao longo da vida: notas para uma reflexão. In A. A. Candeias (Coord.), Desenvolvimento ao Longo da Vida: Aprendizagem, BemEstar e Inclusão (pp. 78-93). Évora: Universidade de Évora (Departamento de Psicologia - Escola de Ciências Sociais). ISBN: 9789898550835

Carapeto, M. J. (2019b). Reorganização do autoconhecimento e adaptação psicológica na adolescência. Revista INFAD de Psicología, International Journal of Developmental and Educational Psychology, 1(2), 137-146.

Coleman, J. C. (2011). The nature of adolescence (4th Ed). New York, NY: Psychology Press.

Durbrow, E. H., Pen, L. F., Masten, A., Sesma, A., \& Williamson, I. (2001). Mothers' conceptions of child competence in contexts of poverty: The Philippines, St Vincent, and the United States. International Journal of Behavioral Development, 25(5), 438-443. 
Diener, E. (2000). Subjective well-being: The science of happiness and a pro-posal for a national index. American Psychologist, 55, 34-43.

Erikson, E (1959). Identity and the Life Cycle. New York, N.Y.: Norton.

Havighurst, R. J. (1948). Developmental tasks and education. University of Chicago Press.

Hollenstein, T., \& Lougheed, J. P. (2013). Beyond storm and stress: Typicality, transactions, timing, and temperament to account for adolescent change. American Psychologist, 68(6), 444.

Inchley, J., \& Currie, D. (2016). Growing up unequal: gender and socioeconomic differences in young people's health and well-being. Health Behaviour in School-aged Children (HBSC) study: international report from the 2013/2014 survey (№ 7). Copenhagen, Denmark: World Health Organization Regional Office for Europe.

Masten, A. S., \& Tellegen, A. (2012). Resilience in developmental psychopathology: Contributions of the project competence longitudinal study. Development and psychopathology, 24(2), 345-361.

McAdams, D. P. (2006). The redemptive self: Generativity and the stories Americans live by. Research in human development, 3(2-3), 81-100.

Meeus, W. (2011). The study of adolescent identity formation 2000-2010: A review of Iongitudinal research. Journal of research on adolescence, 21(1), 75-94.

Sawyer, S. M., Azzopardi, P. S., Wickremarathne, D., \& Patton, G. C. (2018). The age of adolescence. The Lancet Child \& Adolescent Health, 2(3), 223-228.

Smetana, J. G., Robinson, J., \& Rote, W. M. (2014). Socialization in adolescence. In J. E. Grusec and P. D. Hastings (Eds.), Handbook of Socialization: Theory and Research (2nd Ed.) (pp. 60-84). Guilford.

Sousa, B. \& Fonseca, A. C. (2014). Tornar-se adulto em Portugal no início do século XXI: elementos para um retrato. In A. C. Fonseca (Coord.), Jovens adultos (pp. 65-86). Coimbra: Almedina. 
